\title{
The late medical effects of cancer treatments: a growing challenge for all medical professionals
}

\author{
Authors: Jennifer Harrington ${ }^{A}$ and Jeff White ${ }^{B}$
}

\section{Introduction}

2.5 million people live with cancer in the $\mathrm{UK}^{1}$ and, by 2020 , almost half (47\%) of the UK population is expected to get cancer at some point during their lifetime. ${ }^{2}$ With early diagnosis and improving treatments, increasing numbers are living with cancer. However, many are affected by the longterm consequences of cancer therapy. This inaugural 1-day national meeting, organised by the Royal College of Physicians with Macmillan Cancer Support as its principal partner, was designed to highlight the scale of the problem, provide an overview of the systemic effects of cancer treatment and be a forum for discussion of new models of delivering care. The target audience included consultants and trainees from all medical specialties, as well as oncologists and GPs.

\section{Scale of the problem}

While clinicians have largely focused on the initial diagnosis and treatment of cancer, enabling patients to live well after a cancer diagnosis is important. Half of people with cancer may now live for more than 10 years after their diagnosis, yet Macmillan Cancer Support estimate that at least 625,000 people in the UK suffer from chronic medical conditions caused by their cancer or its treatment. ${ }^{3}$ In an ageing population, comorbidities complicate both treatment and recovery, with $70 \%$ of cancer survivors living with one or more potentially serious illnesses. ${ }^{4}$

This conference concentrated on medical side effects, for example the increased risk of other serious conditions such as cardiovascular disease. However, there are many aspects to quality of life for cancer survivors, including addressing social, financial, emotional and practical consequences. The realities of living with cancer were illustrated by Mark Davies, a cancer survivor. His powerful message to clinicians was that they need to not just focus on achieving disease control, but also on survivors' long-term wellbeing.

Authors: ${ }^{\text {A }}$ specialty trainee in medical oncology, Addenbrooke's Hospital, Cambridge, UK; ${ }^{B}$ consultant medical oncologist and honorary clinical senior lecturer, Beatson West of Scotland Cancer Centre, Glasgow, UK

\section{Learning from the paediatrics community}

Two of the main challenges identified were, firstly, raising awareness of the consequences of treatment with both patients and the general medical profession and, secondly, concern about the lack of communication between secondary and primary care at the transition point from active cancer treatment to long-term follow-up. There is an opportunity to learn from research into late effects after childhood cancers, reviewed here by Professor Rod Skinner. Childhood cancer survivor cohort studies have revealed the diversity of 'medical' effects affecting most body systems, including cardiovascular, respiratory, reproductive, endocrine and neurological, and the risk of secondary cancers. ${ }^{5}$ There is increasing concern about the 'concept of frailty' in childhood cancer survivors, which increases the risk of new chronic health conditions. ${ }^{6}$

The overall aim of follow-up is to improve survivors' longterm health by:

1 managing existing late effects

2 active surveillance to look for future side effects, allowing early detection and treatment

3 education of survivors and carers, providing clear information about toxicities, thus promoting engagement and encouraging healthy lifestyle choices.

The model for delivery may vary and the use of risk stratification can help determine how this is best delivered. This can range from low-risk patients largely using selfmanagement in primary care, but with the knowledge of how to access support when needed, to those mediumrisk patients having planned, coordinated follow-up to a group with complex, multidisciplinary needs. A clear transition of care from active cancer treatment to the period beyond, with a detailed treatment summary, is critical. The importance of an individualised survivorship care plan was stressed, making clear when additional screening is required, for example breast screening follow-up after treatment for Hodgkin's lymphoma with radiotherapy, or when repeat echocardiograms are required after cardio-toxic chemotherapy. Evidence-based guidelines exist and are actively being developed with international collaboration for specific topics, such as cardiomyopathy surveillance. $^{7}$ 


\section{Multisystem long-term toxicities of treatment}

It is increasingly likely that all doctors will see patients who have survived cancer and are living with the late effects; such patients may well have been discharged from specialist oncology care. Improving knowledge of side effects and potential consequences is an important part of allowing them to be recognised early, achieving better outcomes and preventing their progression. Short presentations highlighted some of the effects of cancer treatments on:

$>$ bone metabolism

$>$ gastrointestinal system

$>$ metabolic syndrome

$>$ respiratory system

$>$ dermatology

$>$ endocrinology

$>$ cardiology.

With recognition of the scale of the problem, subspecialties such as cardio-oncology are developing. The talk by Dr Andreyev, an onco-gastroenterologist at the Royal Marsden, described how an algorithmic physiological approach to gastrointestinal symptoms can allow appropriate treatments to be identified and impact positively on quality of life. ${ }^{8}$ Other sessions explored chronic pain management, the role of physical activity and the management of cancer related fatigue.

Research in late effects in adults is not as well developed as in survivors of childhood cancers although it is increasing as evidenced in the poster session. One of the first examples of a survivorship randomised controlled trial, looking at testosterone replacement in young men after cancer therapy, was discussed (Cancer Research UK trial number CRUK/10/012). This study demonstrates the need for work across different cancer sites and collaboration with nononcology specialties in order to deliver the most appropriate trial design.

\section{Strategies for survivorship care}

The National Cancer Survivorship Initiative (NCSI) - a partnership between Macmillan Cancer Support, the Department of Health and NHS England - has explored the best way to address these issues in the UK. ${ }^{9}$ Consequences of cancer treatment should be avoided where possible, but when they do occur they need to be identified and addressed. Stratified care pathways, with individualised follow-up, are recommended.

Almost half (42\%) of patients said staff did not fully explain the potential long-term side effects of cancer treatment. ${ }^{10}$ While initial consent may be inadequate (particularly with respect to late effects), it is often difficult for patients to remember everything they are told while they are still trying to come to terms with their diagnosis. Patients need to receive education at the end of each course of treatment on how it will or may affect all aspects of their quality of life in the short, medium and long term.

The importance of a multidisciplinary team (MDT) collaborative approach was highlighted using case studies, with feedback sought from a range of professionals at the meeting and from the audience. The importance of a key worker for patients after treatment was identified, coordinating care and providing a link between primary and secondary care. Resources, such as the Comprehensive Cancer Tool Kit published by Macmillan Cancer Support together with the Royal College of General Practitioners, have been developed. Here, the need for a treatment summary and individualised care plan as developed in paediatrics is crucial, allowing primary care to be aware of potential toxicities and key diagnostic tests required in follow-up. The GPs present at the conference proposed that they could act in this coordinating role if given an appropriate treatment summary.

One new model to deliver survivorship care discussed was the concept of a virtual MDT for patients with complex consequences of cancer treatment, developed by Macmillan Cancer Support in partnership with The Christie Hospital, Manchester. An electronic referral is made to the coordinator, patient details are anonymised and then relevant specialists are contacted and asked to comment, with their feedback collated into a report for the referring clinician. Initial feedback has been positive and it is now in phase II testing.

The NCSI has developed the Recovery Package, ${ }^{9}$ recognised in the NHS England Five Year Forward View and the Cancer Taskforce strategy. This combines four main interventions to improve care coordination and outcomes for cancer patients and should address many of the challenges discussed at the meeting. It consists of:

1 a holistic needs assessment

2 a treatment summary

3 cancer care reviews (conducted by a GP)

4 a health and wellbeing event.

As in paediatrics, there is a need for evidence-based clinical guidelines to support specialists and non-specialists. These are now being developed, and Macmillan Cancer Support are collaborating to produce practical guidelines for both specialists and non-specialists.

\section{Conclusions}

This meeting provided an excellent overview of the emerging health needs of long-term cancer survivors. Early recognition of late medical effects can have a significant impact, improving quality of life and longer-term outcomes. Therefore, raising awareness is critical, educating not just patients but also all healthcare professionals to be alert to potential toxicities. The NCSI has recommended key interventions that should improve current care. However, the implementation of the Recovery Package is a work in progress. Its success will require input from a wide MDT to allow its effective delivery, together with a commitment to ongoing research.

\section{Conflicts of interest}

JW was the chair of the organising committee for this conference.

\section{References}

1 Maddams J, Utley M, Møller H. Projections of cancer prevalence in the United Kingdom, 2010-2040. Br J Cancer 2012:107:1195-202.

2 Macmillan Cancer Support. Cancer mortality trends: 1992-2020. London: Macmillan Cancer Support, 2013. www.macmillan.org.uk/ documents/aboutus/newsroom/mortality-trends-2013-executivesummary-final.pdf [Accessed 24 January 2017]. 
3 Macmillan Cancer Support. Throwing light on the consequences of cancer and its treatment. London: Macmillan Cancer Support, 2013.

4 Macmillan Cancer Support. The burden of cancer and other longterm health conditions. London: Macmillan Cancer Support, 2015. www.macmillan.org.uk/documents/press/cancerandotherlongtermconditions.pdf [Accessed 24 January 2017].

5 Oeffinger KC, Mertens AC, Sklar CA et al. Chronic health conditions in adult survivors of childhood cancer. $N$ Engl J Med 2006:355:1572-82.

6 Ness KK, Krull KR, Jones KE et al. Physiologic frailty as a sign of accelerated aging among adult survivors of childhood cancer: a report from the St Jude Lifetime cohort study. J Clin Oncol 2013;31:4496-503.

7 Armenian SH, Hudson MM, Mulder RL et al. Recommendations for cardiomyopathy surveillance for survivors of childhood cancer: a report from the International Late Effects of Childhood Cancer Guideline Harmonization Group. Lancet Oncol 2015;16:e123-36.
8 Andreyev HJ, Benton BE, Lalji A et al. Algorithm-based management of patients with gastrointestinal symptoms in patients after pelvic radiation treatment (ORBIT): a randomised controlled trial. Lancet 2013;382:2084-92.

9 National Cancer Survivorship Initiative. Living with and beyond cancer: taking action to improve outcomes. London: Department of Health, 2013.

10 Elliott J, Fallows A, Staetsky L et al. The health and well-being of cancer survivors in the UK: findings from a population based survey. Br J Cancer 2011;105 (Suppl 1):S11-20.

Address for correspondence: Dr J Harrington, Cambridge University Hospitals NHS Foundation Trust, Box 193 Addenbrooke's Hospital, Hills Road, Cambridge CB2 0QQ, UK. Email: Jennifer.harrington@addenbrookes.nhs.uk
'This landmark report lays out in the starkest terms yet the devastating impact air pollution is having on our health, our economy and our society as a whole.'

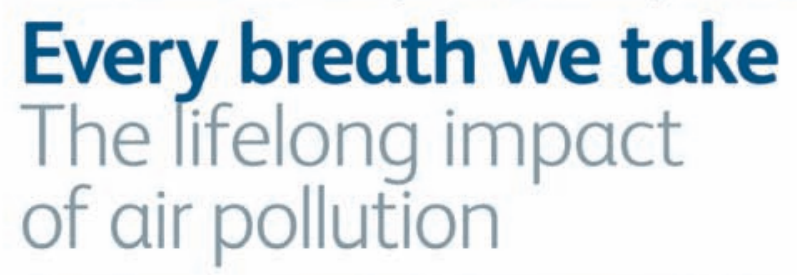

This major report plainly sets out the dangerous impact that air pollution has on our nation's health. Compiled by experts in medicine and environmental sciences, the report discusses the current evidence and draws up recommendations for action.

ISBN 978-1-86016-567-2 £15 including $p+p$ or free to download

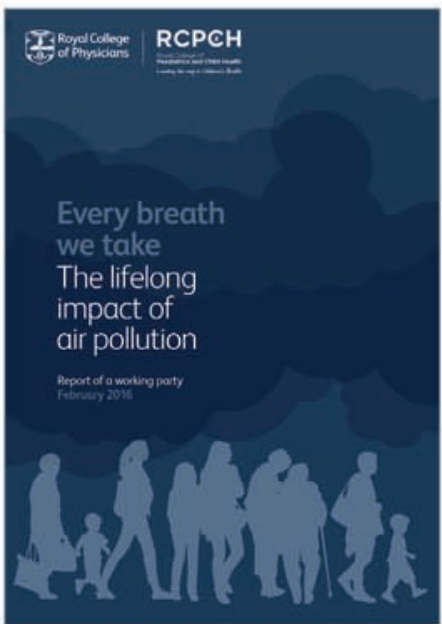

Order a copy: shop.rcplondon.ac.uk 